\title{
A Study of Stellar Model with Cramer's Opacity by using Runge Kutta Method with Programming C
}

\section{Bin Masud A*}

Department of Computer Science and Engineering, Shanto-Mariam University of Creative Technology, Bangladesh

\begin{abstract}
This paper, I have made an investigation on a stellar model with Kramer's Opacity and negligible abundance of heavy elements. I have determined the structure of a star with mass $2.5 \otimes \mathrm{M}$, i.e., the physical variables like pressure, density, temperature and luminosity at different interior points of the star. Discussed about some equation of structure, mechanism of energy production in a star and energy transports in stellar interior and then I solved radiative envelope and convective core by the matching or fitting point method by $\mathrm{C}$ Programming language.
\end{abstract}

Keywords: Energy production in star; Hydrostatic equilibrium of star; Mass conservation; Schwarzschild method and variable; Polytropic core solution; Envelope solution of the matching point

\section{Introduction}

A star is a dense mass that generates its light and heat by nuclear reactions, specifically by the fusion of hydrogen and helium under conditions of enormous temperature and density. Stars are like our sun. The star is powered by hydrogen fusion. The fusion only takes place at core of the star where it is dense enough. The "life" of a star is the time during which it slowly burns up its hydrogen fuel, and evolves only slowly in the process. The star is in force balance between pressure and gravity. It is also in energy balance between production by fusion reactions, transport by photon radiation, and loss from the surface by the (usually) visible radiation by which we can detect the star. The "birth" of a star refers to the process by which it is formed from diffuse clouds of cold gas that are present in its galaxy. A cloud collapses to form a number of stars when it is disturbed so that its gravity overcomes its motion and pressure. The "death" of a star occurs when its fusion fuel, first hydrogen and then heavier nuclei, has run out. This can be very violent if the star is very massive, ending in things like a black hole and/or a supernova, perhaps leaving a neutron star behind. If the star is not very massive, like the Sun or even smaller, it ends by ejecting part of its atmosphere and then settling down to a cold, dense white dwarf. Harm and Schwarzschild (1955) has shown that the maximal possible mass of the star is $60 \mathrm{MA}$ and minimum mass of star is $0.01 M \AA$. The chemical element of star is hydrogen, helium and other heavier elements. If hydrogen, helium and other element were denoted by $\mathrm{X}, \mathrm{Y}$ and $\mathrm{Z}$, respectively. Then $\mathrm{X}+\mathrm{Y}+\mathrm{Z}=1$. For the sun $\mathrm{X}=0.73, \mathrm{Y}=0.25$ and $\mathrm{Z}=0.02$.

\section{Energy Production in Stars}

A normal main sequence star derives energy from its nuclear source. Enormous amount of energy are continually radiated at a steady rate over long spars of time; for example the sun radiates approximately $10^{41}$ ergs per year. Those thermonuclear reactions do produce energy. That a star can derive energy from thermonuclear reaction is understood from the following example, $41 \mathrm{H} 1=2 \mathrm{He} 4+2 \mathrm{~b}++2 v+\gamma$. That means four hydrogen atoms combine to give one helium atom with the production of two positrons $(b+)$, two neutrinos $(v)$ and radiation $(\gamma)$. Energy production mainly in two ways (i) Proton-Proton chain (PP chain) (ii) Carbon-Nitrogen chain (CN chain).

\section{Hydrostatic equilibrium of Star}

Consider a cylinder of mass $\mathrm{dm}$ located at a distance $r$ from centre of the star with height $\mathrm{dr}$ and surface area $\mathrm{A}$ at the top and bottom as shown in Figure 1. Also denote $\mathrm{F}_{\mathrm{p} . \mathrm{t}}$ and $\mathrm{F}_{\mathrm{p.b}}$ to be the pressure forces at the top and bottom of the cylinder respectively If $\mathrm{Fg}<0$ is the gravitational forces on the cylinder then from Newton's second law we have

$$
d m \frac{d^{2} r}{d t^{2}}=F_{g}+F_{p . t}+F_{p . b}
$$

Defining the change in pressure force dFp across the cylinder by

$$
\begin{aligned}
& F_{p . t}=-\left(F_{p . b}+d_{F p}\right) \\
& \text { Then gives } \frac{d^{2} r}{d t^{2}}=F_{g}-d F_{p}
\end{aligned}
$$

The gravitational force on the small mass $d m$ is given by

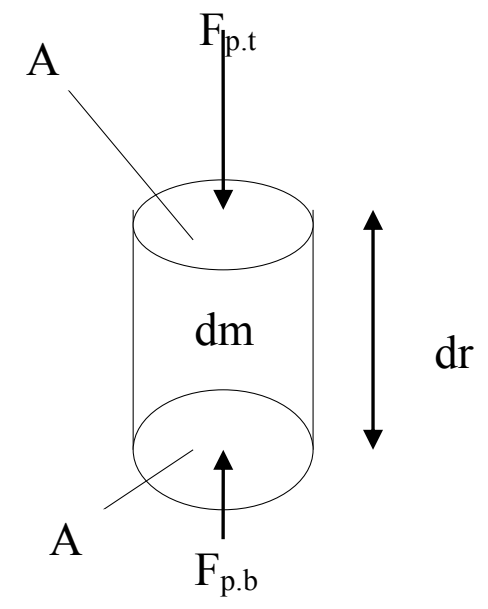

Figure 1: Illustration of hydrostatic equilibrium of tar

*Corresponding author: Bin Masud A, Professor, Department of Computer
Science and Engineering Shanto-Mariam University of Creative Technology Bangladesh, Tel:5456454; E-mail: masud05math@gmail.com

Received June 26, 2017; Accepted July 18, 2017; Published July 21, 2017

Citation: Bin Masud A (2017) A Study of Stellar Model with Cramer's Opacity by using Runge Kutta Method with Programming C. J Phys Math 8: 239. doi: 10.4172/2090-0902.1000239

Copyright: ( 2017 Bin Masud A. This is an open-access article distributed under the terms of the Creative Commons Attribution License, which permits unrestricted use, distribution, and reproduction in any medium, provided the original author and source are credited. 


$$
F_{g}=-G \frac{M(\mathrm{r}) d m}{r^{2}}
$$

From the definition of pressure as the force per unit area we have

$$
P=\frac{F}{A} \Rightarrow d F_{p}=A d p
$$

Putting eqns. (3) and (4) in eqn. (2)

$$
d m \frac{d^{2} r}{d t^{2}}=-G \frac{M(\mathrm{r}) d m}{r^{2}}-A d p
$$

Assuming the density of the cylinder is $r$, then its mass is $\mathrm{dm}=\mathrm{rAdr}$, Now eqn. (5) becomes

$\rho A d r \frac{d^{2} r}{d t^{2}}=-G \frac{M(r) \rho A d r}{r^{2}}-A d p$

Dividing by volume of the cylinder gives

$$
\rho \frac{d^{2} r}{d t^{2}}=-G \frac{M(r) \rho}{r^{2}}-\frac{d P}{d r}
$$

Assuming the star is static the acceleration term will be zero which then leads to

$$
\frac{d P}{d r}=-G \frac{M(r) \rho}{r^{2}}=-\rho g
$$

Where $g=G \frac{M(r)}{r^{2}}$

This is the condition of hydrostatic equilibrium.

\section{Mass Conservation}

Consider a spherically symmetric shell of mass $\mathrm{dMr}$ with thickness $\mathrm{dr}$ and $\mathrm{r}$ is the distance from the centre of the star. The local density is of the shell is $\mathrm{r}$ (Figure 2). The shell's mass is then given by $\mathrm{d} M=\mathrm{d} \operatorname{Vr}(r)$. Since $\delta V=4 \pi r^{2} \delta r$. Then we have $M=4 \pi r^{2} \delta r \rho(r)$

$$
\Rightarrow \frac{d M(r)}{d r}=4 \pi r^{2} \rho(r)
$$

In the limit where $\mathrm{d} \mathrm{r}^{\otimes} 0$ which is the mass conservation equation. Now, the volume of the shell $=4 \mathrm{pr}^{2} \mathrm{dr}$. If $\mathrm{r}$ is the density, then mass of the shell $=4$ pr2rdr. The energy released in the shell can be written as $4 \mathrm{p}$ r2rdre, where e is defined as the energy released per unit mass per unit time. The conservation of energy leads that

$$
\begin{aligned}
& \mathrm{L}_{\mathrm{r}+\mathrm{dr}}-\mathrm{L}_{\mathrm{r}}=4 \pi r^{2} \rho \varepsilon \mathrm{dr} \Rightarrow \frac{d L}{d r}=4 \pi r^{2} \rho \varepsilon \mathrm{dr} \\
& \therefore \frac{d L}{d r}=4 \pi r^{2} \rho \varepsilon
\end{aligned}
$$

\section{Energy Conservation}

Consider a spherical symmetric star in which energy transport is

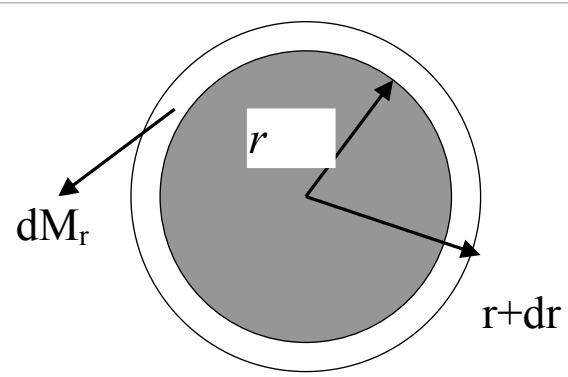

Figure 2: Mass conservation vs. local density is of the shell.

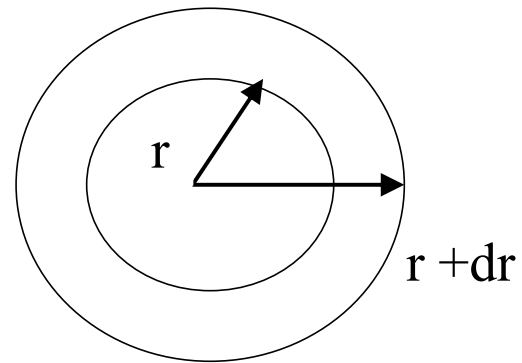

Figure 3: Energy conservation vs. rate of energy flow.

radial in which time variations are very important [1]. Let Lr is the rate of energy flow a across of sphere of radius $\mathrm{r}$ and $\mathrm{L}_{\mathrm{r}+\mathrm{dr}}$ for radius $\mathrm{r}+\mathrm{dr}$.

Now, the volume of the shell $=4 \mathrm{pr}^{2} \mathrm{dr}$. If $\mathrm{r}$ is the density, then mass of the shell is illustrated in Figure 3.

$=4 \mathrm{p} \mathrm{r} 2 \mathrm{rdr}$.

The energy released in the shell can be written as $4 \mathrm{pr}^{2} \mathrm{rdre}$, where $e$ is defined as the energy released per unit mass per unit time. The conservation of energy leads that

$$
\begin{aligned}
& \mathrm{L}_{\mathrm{r}+\mathrm{dr}}-\mathrm{L}_{\mathrm{r}}=4 \pi r^{2} \rho \varepsilon \mathrm{dr} \\
& \Rightarrow \frac{d L}{d r}=4 \pi r^{2} \rho \varepsilon \mathrm{dr} \\
& \therefore \frac{d L}{d r}=4 \pi r^{2} \rho \varepsilon
\end{aligned}
$$

This is the equation of energy conservation.

\section{Energy Transport in Stellar Interior}

Energy transport in stellar interiors occurs by three mechanisms, i.e., radiation, convection and conduction.

\section{Radiation}

Photons carry energy but constantly interact with electrons and ions. Each interaction causes the photon, on average, to lose energy to the plasma. $\Rightarrow$ Increase in gas temperature.

\section{Convection}

Energy is carried by macroscopic mass motion (rising gas) though there is no net mass flux. If the density of an element of gas is less than that of its surroundings, it rises $\Rightarrow$ Schwarzschild criterion for convection [2].

\section{Conduction}

Energy is carried by mobile electrons, which collide with ions and other electrons, but still make progress through the star. The diffusive nature of this process makes it describable in a way similar to radiative transport.

\section{Radiative energy transport}

If the condition of the occurrence of convection is failed then radiative transfer occurs. The energy carried by radiation per square meter per second i.e., flux Frad can be expressed in terms of the temperature gradient and a coefficient of radiative conductively lrad as follows

$$
\mathrm{F}_{\mathrm{rad}}=-\lambda_{\mathrm{rad}} \frac{\mathrm{dT}}{\mathrm{dr}}
$$


Where -ve sign indicates that heat flows down the temperature gradient. Assuming that all energy is transported by radiation. We will now drop the suffix rad,

$$
\therefore F=-\lambda \frac{\mathrm{dT}}{\mathrm{dr}}
$$

Astronomers prefers to work with an inverse of the conductivity known as opacity which opacity

$$
\kappa=\frac{4 a c T^{3}}{3 \rho \lambda}
$$

Where $a$ is the radiation density constant, $\mathrm{c}$ is the speed of light.

From eqn. (12) we have $\lambda=\frac{4 a c T^{3}}{3 \rho \kappa}$

Putting eqns. (13) in eqn. (11) we have

$$
F=-\frac{4 a c T^{3}}{3 \kappa p} \frac{d T}{d r}
$$

We know flux and luminosity equation is

$$
\begin{aligned}
& L=4 \pi r^{2} \mathrm{~F} \\
& \mathrm{~L}=-\frac{16 a c \pi r^{2} T^{3}}{3 \kappa \rho} \frac{d T}{d r} \text { from eqn. (14) } \\
& \Rightarrow \frac{d T}{d r}=\frac{3 \kappa \rho}{6 a c \pi r^{2} T^{3}}
\end{aligned}
$$

This equation is known as the equation of radiative transfer.

\section{Convective Energy Transport}

Let $\rho_{1}^{*}$ and $P_{1}^{*}$ be the density and pressure inside the blob in its original position, the corresponding quantities outside being $\mathrm{r} 1$ and $\mathrm{P} 1$. In its displaced position, let $\rho_{2}^{*}$ and $P_{2}^{*}$ be the density and pressure inside the blob white corresponding quantities outside be $\mathrm{r}_{2}$ and P2. Before the perturbation, $\rho_{1}^{*}=\mathrm{r}_{1}$ and $P_{1}^{*}=P_{1}$ after the perturbation

$$
\rho_{2}^{*}=\rho_{1}^{*}\left(\frac{P_{2}^{*}}{P_{1}^{*}}\right) \text { and } P_{2}^{*}=P_{2}
$$

Where $\mathrm{g}$ is the ratio of specific that $\frac{C_{p}}{C_{v}}$ and has the value $5 / 3$ for highly ionized gas. The layer may be stable if $\rho_{2}^{*}>\rho_{2}$. Therefore mass motion will occur if $\rho_{2}^{*}<\rho_{2}$. Now we have from the above equations $\rho_{2}^{*}=\rho_{1}^{*}\left(\frac{P_{2}}{P_{1}}\right)^{1 / \gamma}$

The equilibrium is stable if $\rho_{1}\left(\frac{P_{2}}{P_{1}}\right)^{1 / \gamma}>\rho_{2}$

And the equilibrium is unstable if $\rho_{1}(-)^{1 / 1} \quad \rho_{2}$

Let $\mathrm{P}_{1}=\mathrm{P}(\mathrm{r})$ andr $\mathrm{r}_{1}=\mathrm{r}(\mathrm{r}) \mathrm{P}_{2}=\mathrm{P}(\mathrm{r}+\mathrm{dr})$ and $\mathrm{r}_{2}=\mathrm{r}(\mathrm{r}+\mathrm{dr}) d r$

From stable condition we have $\left(\frac{P_{2}}{P_{1}}\right)^{1 / \gamma}>\frac{\rho_{2}}{\rho_{1}}$

From unstable condition we have $\left(\frac{P_{2}}{P_{1}}\right)^{1 / \gamma}<\frac{\rho_{2}}{\rho_{1}}$

which implies

$$
\left(\frac{P(r+d r)}{P(r)}\right)^{l / \gamma}>\text { or }<\frac{\rho(r+d r)}{\rho(r)}
$$

Or

$$
\left(1+\frac{1}{P} \frac{d P}{d r} d r\right)^{1 / r}>\text { or }<\left(1+\frac{1}{P} \frac{d P}{d r} d r\right)
$$

Expanding left side of the above inequalities in Taylor series and neglecting higher order terms

$$
\begin{aligned}
& \text { we have }\left(1+\frac{1}{\lambda P} \frac{d P}{d r} d r\right)^{1 / r}>\text { or }<\left(1+\frac{1}{\rho} \frac{d \rho}{d r} d r\right) \\
& \Rightarrow \frac{1}{\gamma P} \frac{d P}{d r}>\text { or }<\frac{1}{\rho} \frac{d \rho}{d r}
\end{aligned}
$$

We know

$P=\frac{K}{\mu H} \rho^{T}$

Taking log and differentiating we have

$$
\frac{1}{P} \frac{d P}{d r}=\frac{1}{\rho} \frac{d \rho}{d r}+\frac{1}{T} \frac{d T}{d r}
$$

For stability condition we have

$$
\begin{aligned}
& \frac{1}{\gamma P} \frac{d P}{d r}>\frac{1}{P} \frac{d P}{d r}-\frac{1}{T} \frac{d T}{d r} \\
& \Rightarrow-\left(1-\frac{1}{\gamma}\right) \frac{d P}{d r}>-\frac{P}{T} \frac{d T}{d r}
\end{aligned}
$$

Therefore mass motion will occur when

$$
-\left(1-\frac{1}{\gamma}\right) \frac{d P}{d r}<-\frac{P}{T} \frac{d T}{d r}
$$

Schwarzschild (1958) has shown that the temperature gradient for the convection is well represented by

$$
\frac{d T}{d r}=\left(1-\frac{1}{\gamma}\right) \frac{T}{P} \frac{d P}{d T}
$$

which is known as convective energy transport equation.

\section{Schwarzschild Method and Variable}

When one is searching for the numerical solution to a physical problem, it is convenient to re-express the problem in terms of a set of dimensionless variables whose range is known and conveniently limited. This is exactly what the Schwarzschild variables accomplish [3]. Define the following set of dimensionless variables

$$
\begin{aligned}
& x=\frac{r}{R} \\
& q=\frac{M(r)}{M} \\
& l=\frac{L(r)}{L} \\
& p=P(\mathrm{r}) \frac{4 \pi \mathrm{R}^{4}}{G M^{2}} \\
& t=T(\mathrm{r}) \frac{R}{\mu} \frac{R}{G M} \\
& \rho(r)=\frac{M}{4 \pi R^{3}} \frac{p}{t}
\end{aligned}
$$

Note that the first three variables are the fractional radius, mass 
and luminosity, respectively and after three variables represented the pressure, temperature and density. In addition, let us assume that the opacity and energy generation rate can be approximately by

and $\kappa=\kappa_{0}\left(\frac{\rho}{T^{3.5}}\right), \varepsilon p p=\varepsilon_{0} \rho X X_{C N} \times\left(\frac{T}{10^{6}}\right)^{16}$

where $\mathrm{k}_{0}=4.34 \times 10^{25} \times \mathrm{Z}(1+\mathrm{X})$

Putting eqns. (17), (18), (20) and (19) in eqn. (6), we have

$\frac{d}{d(R x)}\left(\frac{G M^{2} p}{4 \pi R^{4}}\right)=-\frac{G q M}{R^{2} x^{2}} \frac{M}{4 \pi R^{3}} \frac{p}{t}$

Again, putting eqns. (17), (18) and (22) in eqn. (7), we have

$\Rightarrow \frac{G M^{2}}{4 \pi R^{5}} \frac{d p}{d x}=-\frac{G M^{2}}{4 \pi R^{5}} \frac{p q}{t x^{2}}$

$\Rightarrow \frac{d p}{d x}=-\frac{p q}{t x^{2}}$

Again, putting eqns. (17), (18) and (22) in eqn. (7), we have

$\frac{d}{d(x R)}(q M)=4 \pi R^{2} x^{2} \frac{M}{4 \pi R^{3}} \frac{p}{t}$

$\Rightarrow \frac{M}{R} \frac{d q}{d x}=\frac{M}{R} \frac{p x^{2}}{t}$

$\Rightarrow \frac{d q}{d x}=\frac{p x^{2}}{t}$

Now putting eqns. (17), (19), (22) and (21) in eqn. (9), we have

$\frac{d}{d(x R)}(l L)=4 \pi R^{2} x^{2} \varepsilon_{0} \rho^{2} X X_{C N} \times\left(\frac{T}{10^{6}}\right) 16$

$\frac{L}{R} \frac{d l}{d x}=\varepsilon_{0} X X_{C N}\left(\frac{M}{4 \pi R^{3}} \frac{p}{t}\right)^{2} \frac{1}{10^{96}}\left(\frac{t M}{R}\right)^{16} \times(\mu \mathrm{G}) \times\left(\frac{1}{R}\right)^{16}$

$\left.\Rightarrow \frac{d l}{d x}=\frac{\varepsilon_{0} X X_{C N}}{4 \times 10^{96} \pi} \mu \mathrm{G}\right) \cdot\left(\frac{1}{R}\right)^{16} \cdot \frac{M^{18}}{L R^{19}} \cdot p^{2} x^{2} t^{14}$

$\Rightarrow \frac{d l}{d x}=D p^{2} x^{2} t^{14}$

Where $D=\frac{\varepsilon_{0} X X_{C N}}{4 \times 10^{96} \pi} \times(\mu \mathrm{G}) \times\left(\frac{1}{R}\right)^{16} \times \frac{M^{18}}{L R^{19}}$ get

Again putting eqns. (17), (19), (21), (22) and (23) in eqn. (15), we

$$
\begin{aligned}
& D=\frac{\varepsilon_{0} X X_{C N}}{4 \times 10^{96} \pi} \times(\mu \mathrm{G}) \times\left(\frac{1}{R}\right)^{16} \times \frac{M^{18}}{L R^{19}} \\
& \frac{d}{d(R x)}\left(\frac{G M t}{R}\right) \frac{\mu}{R}=\frac{3 \kappa_{0} l L}{16 \pi a c R^{2} x^{2}} \times \frac{\rho^{2}}{T^{6.5}} \\
& \Rightarrow \frac{\mu}{R} G M \frac{d t}{d x} \\
& =-\frac{3 \kappa_{0} l L}{16 \pi a c R^{2} x^{2}} \times\left(\frac{M}{4 \pi R^{3} t} \frac{p}{t}\right)^{2} \times\left(\frac{R}{G M t}\right)^{6.5} \times\left(\frac{R}{\mu}\right)^{6.5} \\
& \Rightarrow \frac{d t}{d x}=-\frac{3 \kappa_{0}}{256 \pi^{3} a c}\left(\frac{1}{G}\right)^{7.5} \frac{L R^{0.5}}{M^{5.5}} \frac{p^{2} l}{x^{2} t^{8.5}} \\
& \Rightarrow \frac{d t}{d x}=-C \frac{p^{2} l}{x^{2} t^{8.5}}
\end{aligned}
$$

Where $C=\frac{3 \kappa_{0}}{256 \pi^{3} a c}\left(\frac{1}{G}\right)^{7.5} \frac{L R^{0.5}}{M^{5.5}}$

Putting eqns. (17), (19) and (20) in eqn. (16) we have

$\frac{d t}{d x}=\frac{2}{5} \frac{t}{p} \frac{d p}{d x}$

Finally we have the full set equations

$$
\begin{aligned}
\frac{d q}{d x} & =\frac{-p x^{2}}{t} \\
\frac{d q}{d x} & =\frac{-p x^{2}}{t} \\
\frac{d l}{d x} & =D p^{2} x^{2} t^{14} \\
\frac{d t}{d x} & =-C \frac{p^{2} l}{x^{2} t^{8.5}} \text { (Radiative layer) } \\
\frac{d t}{d x} & =\frac{2}{5} \frac{t}{p} \frac{d p}{d x} \text { convective layer }
\end{aligned}
$$

which are subject to the boundary conditions

$$
q(0)=l(0)=0 \text { and } q(1)=l(1)=1 .
$$

If the star has a convective core, then all the energy is produced in a region where the structure is essentially specified by the adiabatic gradient and so the energy conservation equation (29) is redundant. This means that the $\mathrm{D}$ is unspecified and the problem will be solved by determining $\mathrm{C}$ alone. Such a model is known as a Cowling model [4].

\section{Solution of the Model}

Since the model star is likely to have a small convective core with a radiative envelope, in principle we have two solutions, one is the envelope and another is the core. The two solutions must match at the interface.

a) Polytropic core solution

b) Envelope solution

\section{Polytropic core solution}

Eliminating q from eqns. (27) and (28) we have

$$
\begin{aligned}
& \frac{d}{d x}\left(-\frac{t x^{2}}{p} \frac{d p}{d x}\right)=\frac{p x^{2}}{t} \Rightarrow \frac{d}{d x}\left(-\frac{t x^{2}}{p} \frac{p}{t} \frac{d t}{d x}\right)=\frac{p x^{2}}{t} \\
& \Rightarrow-\frac{d}{d x}\left(-\frac{d t}{d x}\right)=-\frac{p x}{t}
\end{aligned}
$$

Now introducing the polytropic variables $\mathrm{h}$ and $\mathrm{q}$, defined by

$$
p=p c \theta^{5 / 2} t=t_{c} \theta, x=\left(\frac{5}{2} \frac{t_{c}^{2}}{p_{c}}\right)^{1 / 2} \eta
$$

Where $\mathrm{pc}$ and tc are the central pressure and temperature in nondimensional. Now putting the value of $\mathrm{p}, \mathrm{t}$ and $\mathrm{x}$ in (3.4), we have

$$
\begin{aligned}
& \frac{5}{2}\left(\frac{2 p_{c}}{5 t_{c}^{2}}\right)^{1 / 2} \frac{d}{d \eta}\left(\frac{5}{2} \frac{t_{c}^{2}}{p_{c}} \eta^{2}\left(\frac{2 p_{c}}{5 t_{c}^{2}}\right)^{1 / 2} t c \frac{d \theta}{d \eta}\right)=\frac{5 p c \theta^{5 / 2}}{2} \frac{t_{c}^{2}}{p c} \eta^{2} \\
& \Rightarrow \frac{5}{2} \frac{2 p_{c}}{5 t_{c}^{2}} \frac{t_{c}^{3}}{p_{c}} \frac{d}{d \eta}\left(\eta^{2} \frac{d \theta}{d \eta}\right)=-\eta^{2} t_{c} \theta^{3 / 2}
\end{aligned}
$$


$\Rightarrow \frac{1}{\eta^{2}} \frac{d \theta}{d \eta}\left(\eta^{2} \frac{d \theta}{d \eta}\right)=-\theta^{3 / 2}$

Which is the Lane-Emden equation with index

$n=\frac{3}{2}$

The general solution eqn. (34) is

$\theta=1-\frac{1}{6} \eta^{2}+\frac{3 / 2}{120} \eta^{4}-\frac{3 / 2(8 \times 3 / 2-5)}{42 \times 360} \eta^{6}+\ldots .$.

$\Rightarrow \theta=1-\frac{1}{6} \eta^{2}+\frac{1}{80} \eta^{4}-\frac{1}{1440} \eta^{6}+\ldots \ldots$

For small $\mathrm{h}$ this is a rapidly convergent series. We take $\theta=1-\frac{1}{6} \eta^{2}+\frac{1}{80} \eta^{4}$

Introducing Schwarz child homology variables defined by

$U=d \frac{\ln M(\mathrm{r})}{d \ln r}=\frac{r}{M(\mathrm{r})} \frac{d M(\mathrm{r})}{d r}$

$=\frac{x R}{q M} \frac{M d q}{R d x}$

$=\frac{x}{q} \frac{d q}{d x}$

$=-\frac{x}{\frac{t x^{2}}{p} \frac{d p}{d x}} \cdot \frac{p x^{2}}{t}$

$=-\frac{p^{2} x}{t^{2} \frac{d p}{d x}}$

$=\frac{-p^{2} x}{t^{2} \frac{5 p}{2 t} \frac{d t}{d x}}$

$=-\frac{2}{5} \frac{p x}{t} \frac{1}{\frac{d t}{d x}}$

$=\frac{2}{5} \frac{p_{c} \theta^{5 / 2}}{t_{c} \theta}\left(\frac{5}{2} \frac{t_{c}^{2}}{p_{c}}\right)^{1 / 2} \eta \frac{1}{\frac{t_{c}}{\left(\frac{5}{2} \frac{t_{c}^{2}}{p_{c}}\right)^{1 / 2}} \frac{d \theta}{d \eta}}$

$=\frac{2}{5} \frac{p_{c}}{t_{c}^{2} \theta} \theta^{3 / 2} \frac{5 t_{c}^{2}}{2 p_{c}} \eta \frac{1}{\frac{d \theta}{d \eta}}$

$=-\theta^{3 / 2} \eta \frac{1}{\frac{d \theta}{d \eta}}$

$\cong 3-\frac{3 \eta^{2}}{10}+\ldots$

and $V \equiv-\frac{d \ln \mathrm{P}}{d \ln r}=-\frac{r}{p} \frac{d P}{d r}$

$=\frac{x R}{G M^{2}} \frac{G M^{2}}{4 \pi R^{4}} \frac{d p}{R d x}$

$\frac{G M^{2}}{4 \pi R^{4}} 4 \pi R^{4} R d x$

$=\frac{x}{p} \frac{d p}{d x}$

$-\frac{\left(\frac{5}{2} \frac{t_{c}^{2}}{p_{c}}\right)^{1 / 2} \eta}{p c \theta^{5 / 2}} \frac{d \theta^{5 / 2}}{\left(\frac{5}{2} \frac{t_{c}^{2}}{p_{c}}\right) d \eta}$

$=-\frac{\eta}{\theta^{5 / 2}} \frac{d \theta^{5 / 2}}{d \eta} \cong \frac{5 \eta^{2}}{6}\left(1+\frac{n^{2}}{60}+\ldots\right)$
So as to good approximation $U=3-\frac{18}{50} \mathrm{~V}$

This gives the core solution in the $\mathrm{U}-\mathrm{V}$ plane.

\section{Envelope solution of the matching point}

The envelope of the model star is radiative equilibrium. This structure is determined by equations (27), (28) and (30). The equation (30) contains an unknown parameter C. Our aim is to determine the correct value of $\mathrm{C}$ and obtain the envelope solution for the value of the parameter. In order to do this we have to solve the envelope solutions for different trial value of $\mathrm{C}$ and find which value of $\mathrm{C}$ the solution just matches the core solution at the interface. However the solution is not straightforward. Because of the existence of singularity at the surface, integration cannot be started right from the surface $(\mathrm{x}=\mathrm{l})$. To avoid this difficulty we have to look for series expansion of the variables about the singular point. The envelope solutions we have calculated numerically, however since the equations are singular at the surface, $\mathrm{p}=\mathrm{t}=0$. We have chosen the series expansion of the variables near the singular point in the following way $[1,3]$. Let

$$
\begin{aligned}
& \frac{1}{x}-1=\xi \Rightarrow d x=-x^{2} d \xi \\
& \therefore \frac{d p}{d x}=-\frac{q p}{t x^{2}} \Rightarrow \frac{d p}{-x^{2} d \xi}=\frac{q p}{t x^{2}} \Rightarrow \frac{d p}{d \xi}=\frac{q p}{t} \\
& \Rightarrow t \frac{d p}{d \xi}=q p \\
& \frac{d t}{d x}={ }_{-} C \frac{p 2}{t^{8.5} x^{2}} \Rightarrow \frac{d t}{-x^{2} d \xi}=-C \frac{p^{2}}{t^{8.5} x^{2}} \\
& \Rightarrow \frac{d t}{d \xi}=C \frac{p^{2}}{t^{8.5}} \Rightarrow t^{8.5} \frac{d t}{d \xi}=C p^{2} \\
& \text { And } \frac{d p}{d x}=\frac{p x^{2}}{t} \Rightarrow \frac{d q}{-x^{2} d \xi}=\frac{p x^{2}}{t} \Rightarrow \frac{d q}{d \xi}=-\frac{p x^{4}}{t} \\
& \Rightarrow \frac{d q}{d \xi}=\frac{P}{t(1+\xi)^{4}}
\end{aligned}
$$

Here the singular point is $\mathrm{x}=0$, since $\mathrm{x}=1$ i.e., $\mathrm{x}=0$. Now the series expansion of variables about $\mathrm{x}=0$ can be easily done. By Fuchs theorem, a convergent development of the solution in a power series about the singular point having a finite number of terms is possible. We therefore take

$$
\begin{aligned}
& t=\xi^{u}\left(a_{0}+a_{1} \xi+\ldots .+a_{\mathrm{n}} \xi^{\mathrm{n}}\right) \\
& p=\xi^{v}\left(b_{0}+b_{1} \xi+\ldots .+b_{\mathrm{n}} \xi^{\mathrm{n}}\right)
\end{aligned}
$$

And $\mathrm{q}=1+\mathrm{c}_{1} \xi+c_{2} \xi+\ldots+c_{n} \xi^{\mathrm{n}}$

We have

$$
\begin{aligned}
& \xi^{u}\left(a_{0}+a_{1} \xi+\ldots .+a_{\mathrm{n}} \xi^{\mathrm{n}}\right) \frac{d}{d \xi}\left\{\xi^{v}\left(b_{0}+b_{1} \xi+\ldots .+b_{\mathrm{n}} \xi^{\mathrm{n}}\right)\right\} \\
& =\left(1+\mathrm{c}_{1} \xi+c_{2} \xi+\ldots+c_{n} \xi^{\mathrm{n}}\right)\left\{\xi^{v}\left(b_{0}+b_{1} \xi+\ldots .+b_{\mathrm{n}} \xi^{\mathrm{n}}\right)\right\} \\
& \Rightarrow a_{0} b_{0} v \xi^{u+v-1}+\left(a_{1} b_{0} v+a_{0} b_{1}\right) \xi^{u+v}+\ldots .=b_{0} \xi^{v}+\left(b_{1}+b_{0}+c_{1}\right) \xi^{v+1}+\ldots
\end{aligned}
$$

Since the two polynomial are equal must have

$u+v-1=v$ and $u+v=v+1$ and $a_{0} b_{0} v=b_{0}$

$u=1$ and $a_{0} v=1$.

we have 


$$
\begin{aligned}
& t=a_{0} \zeta+a_{1} \zeta^{2}+\ldots . .+a_{n} \zeta^{n+1} \\
& \therefore\left(\mathrm{a}_{0} \xi+\mathrm{a} 1 \xi 2+\ldots+a n \xi n+1\right) 8.5 \frac{d}{d \xi}\left(\mathrm{a}_{0} \xi+a_{1} \xi 2+\ldots a_{n} \xi n+1\right) \\
& =\mathrm{C}\left(\mathrm{b}_{0} \xi+b_{1} \xi^{v+1} . . b n \xi^{v+n}\right)^{2} \\
& \Rightarrow\left\{\left(a_{0} \xi\right)^{8.5}+8.5\left(\mathrm{a}_{0} \xi\right)^{7.5}\left(a_{1} \xi^{2}+\ldots+a n \xi^{n+1}\right)+\ldots\right\} \mathrm{a}_{0}+2 \mathrm{a}_{1} \xi+\ldots \\
& =C\left\{\mathrm{~b}_{0}^{2} \xi^{2 v}+\left(2 \mathrm{~b}_{0} \mathrm{~b}_{1}\right) \xi^{2 v+1}+\ldots\right\}
\end{aligned}
$$

Again equating the powers and coefficients we

have

$$
\begin{aligned}
& 2 \mathrm{v}=8.5 \text { and } c b_{0}^{2}=a_{0}^{9.5} \\
& \mathrm{v}=4.25 \text { and } a_{0}=\frac{1}{4.25}
\end{aligned}
$$

Again we have $b_{0}=\frac{1}{C^{0.5}}\left(\frac{1}{4.25}\right)^{4.75}$

Therefore, in the first approximation we have about $\xi=0$ i.e., $x=1$

$$
\begin{aligned}
& p \approx \xi^{v} b_{0} \\
& =\xi^{4.25} \frac{1}{C^{0.5}}\left(\frac{1}{4.25}\right)^{4.75} \\
& =\frac{1}{C^{0.5}}\left(\frac{1}{4.25}\right)^{4.75}\left(\frac{1}{x}-1\right)^{4.75} \\
& t \approx \xi^{u} a_{0} \\
& =\xi \frac{1}{4.25} \\
& =\frac{1}{4.25}\left(\frac{1}{x}-1\right)
\end{aligned}
$$

\section{And $\mathrm{q} \approx 1$.}

These relations determine the values of the parameters at any point near the surface. With these values as the boundary values the envelope equations can easily be solved numerically for given of C. C is an unknown constant whose value for a start of given mass depends on its luminosity and radius. For solar type stars $C$ is of the order of $10^{-6}$. We shall treat $\mathrm{C}$ as a free parameter and consider of values of close to $10^{-6}$. We take a point $\mathrm{x}=0.99$ very near to the surface. Appropriate for convection, by the fourth order Runge-Kutta method for a number of trial values of $\mathrm{C}$. Some of these calculations, namely for $\mathrm{C}=1.56 \mathrm{e}^{-6}$ , $C=5.6 \mathrm{e}^{-7} \mathrm{C}=9.46 \mathrm{e}^{-7}$. Together with the convective track, equation (36), are drawn in the (U-V) plane (Figure 3 ) at the junction between the convective core and the radiative envelope both $(\mathrm{U}, \mathrm{V})$ and their derivatives must be continuous. So the curve for the correct radiative solution must touch the convective curve at the interface. Form Figure 4 it is found that this happens for $C=9.46 \mathrm{e}^{-7}$. This is the correct value of C for our model star.

Then from equation (6) the values of the parameters that point are found to be.

$$
\begin{aligned}
& p_{0}=3.5136 \times 10^{-9} \\
& t_{0}=2.3767 \times 10^{-3} \\
& q_{0}=1
\end{aligned} .
$$

Taking these values as the boundary values we have integrated the equations for the radiative envelop numerically inwards up to where

$$
0.168 \leq x<1
$$

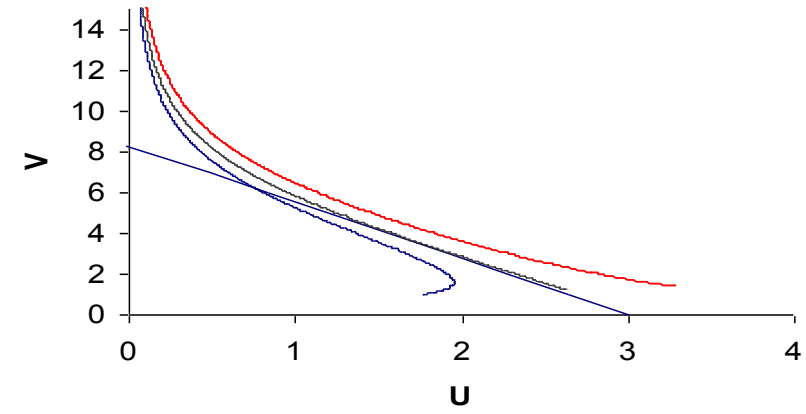

Figure 4: The core solution and the envelope solutions with different values of $\mathrm{C}$ in the UV plane.

For this value of $\mathrm{C}$ the matching point is at $x_{f}=0.168$ The radiative solution for the envelop is $0.168 \leq x<1$ for $\mathrm{c}=9.46 \mathrm{e}^{-7}$ is given in Table 1

Radiative structure of the model star $\mathrm{M}=2.5, \mathrm{X}=0.90, \mathrm{Y}=0.09$, $\mathrm{Z}=0.01$ (solar Unit).

\section{Core Solution of the Model}

From the Table 1 we find that

$$
p_{f}=57.5 q_{f}=0.147 t_{f}=0.708 U_{\mathrm{f}}=2.6253 v_{\mathrm{f}}=1.2323 \text { also } l_{f}=1 \text { at } x=x_{f}
$$

Since all the energy is produced in the core. With these values as our boundary conditions we have to solve the core equations, namely equations (27), (28), (29) and (31) inwards numerically. In order to do this we need the correct value of $\mathrm{D}$. This can be done by integrating the equation. Total luminosity,

$$
\begin{aligned}
& L=\int_{0}^{r f} 4 \pi r^{2} \rho(\mathrm{r}) \varepsilon \mathrm{dr} \\
& =\int_{0}^{x j} 4 \pi r^{2} \rho(\mathrm{r}) \varepsilon_{0} \rho X X_{C N}\left(\frac{T}{10^{6}}\right) R d x \\
& =\int_{0}^{x f} 4 \pi x^{2} R^{2}\left(\frac{M}{4 \pi R^{3}} \frac{p}{t}\right)^{2} X X_{C N} \frac{1}{10^{96}}(\mu \mathrm{G})^{16}\left(\frac{1}{R}\right)^{16} \cdot\left(\frac{G M}{R} t\right)^{16} R d x \\
& \left.=\frac{\varepsilon_{0} X X_{C N}}{4 \times 10^{96} \pi} \mu \mathrm{G}\right) \cdot\left(\frac{1}{R}\right)^{16} \cdot \frac{M^{18}}{R^{19}} \cdot \int_{0}^{x f} x^{2} p^{2} t^{14} d x \\
& =L D \int_{0}^{x f} x^{2} p^{2} t^{14} d x \\
& \therefore \frac{1}{D}=\int_{0}^{x f} x^{2} p^{2} t^{14} d x \\
& =\int_{0}^{n f}\left(\frac{5}{2} \frac{t_{c}^{2}}{p_{c}} \eta^{2} p_{c}^{2} \theta^{5} t_{c}^{14} \theta^{14}\left(\frac{5}{2}\right)^{1 / 2} \frac{t c}{p_{c}^{1 / 2}} d \eta\right) \\
& \int_{0}^{n f}\left(\frac{5}{2}\right)^{1.5} p_{c}^{0.5} t_{c}^{17} \eta^{2} \theta^{19} d \eta \\
& =\left(\frac{5}{2}\right)^{1.5} p_{c}^{0.5} t_{c}^{17} \int_{0}^{n f} \eta^{2}\left(1-\frac{1}{6} \eta^{2}+\frac{1}{40} \eta^{4}-\ldots\right)^{19} d \eta
\end{aligned}
$$

Since $\mathrm{p}$ and $\mathrm{t}$ are continuous at $\mathrm{xf}$

$$
p_{f}=p_{c} \theta^{5 / 2} \text { and } t_{f}=t_{c} \theta_{f}
$$

And also we have 
Citation: Bin Masud A (2017) A Study of Stellar Model with Cramer's Opacity by using Runge Kutta Method with Programming C. J Phys Math 8: 239. doi: 10.4172/2090-0902.1000239

Page 7 of 8

\begin{tabular}{|c|c|c|c|c|c|c|}
\hline $\mathbf{x}$ & $\mathbf{P}$ & $q=M r / M$ & $\mathbf{T}$ & $\mathbf{p}$ & $\log p$ & 1=Lr/L \\
\hline & $1.00 \mathrm{E}+000.00 \mathrm{E}+001.00 \mathrm{E}+00$ & & $0.00 \mathrm{E}+00$ & & & \\
\hline 1.E+00 & & 3.51E-091.00E+00 & 2.E-03 & 5.E-07 & $-6.290871 .00 \mathrm{E}$ & +00 \\
\hline 1.E+00 & & 4.24E-081.00E +00 & 4.E-03 & 3.E-06 & $-5.469721 .00 \mathrm{E}$ & +00 \\
\hline \multirow[t]{8}{*}{ 1.E+00 } & & 2.32E-071.00E+00 & 6.E-03 & 1.E-05 & $4.904341 .00 \mathrm{E}$ & +00 \\
\hline & 1.74E-015.50E+01 & 2.E-01 & & 6.96E-012.74E+011438158 & & $1.00 \mathrm{E}+00$ \\
\hline & 1.73E-015.54E+01 & 2.E-01 & & 6.98E-012.75E+011.440059 & & $1.00 \mathrm{E}+00$ \\
\hline & $1.72 \mathrm{E}-015.58 \mathrm{E}+01$ & 2.E-01 & & 7.00E-012.77E+011.441941 & & $1.00 \mathrm{E}+00$ \\
\hline & 1.71E-015.62E+01 & 2.E-01 & & 7.02E-012.78E+011443804 & & $1.00 \mathrm{E}+00$ \\
\hline & 1.70E-015.67E+01 & 2.E-01 & & 7.04E-012.80E+011.446415 & & $1.00 \mathrm{E}+00$ \\
\hline & 1.69E-015.71E+01 & $1.49 \mathrm{E}+01$ & & 7.06E-012.81E+011.448236 & & $1.00 \mathrm{E}+00$ \\
\hline & 1.68E-015.75E+01 & 1.E-01 & & 7.08E-012.82E+011450039 & & $1.00 \mathrm{E}+00$ \\
\hline
\end{tabular}

Table 1: Radiative structure of the model star $M=2.5, X=0.90, Y=0.09, Z=0.01$ (solar unit).

\begin{tabular}{|c|c|c|c|c|c|c|}
\hline $\mathbf{x}$ & $\mathbf{P}$ & $\mathbf{q}$ & $\mathbf{t}$ & $\mathbf{p}$ & $\log p$ & L \\
\hline \multirow[t]{6}{*}{ 2.E-01 } & $6 . E+01$ & 1.44E-017.10E- & & $128.30 \mathrm{E}+00$ & 1.0 & 1.E+00 \\
\hline & 1.66E-015.84E+01 & 1.42E-017.12E- & & 128.50E+00 & 1454337 & 1.E+00 \\
\hline & 1.65E-015.88E+01 & 1.40E-017.15E- & & $128.50 \mathrm{E}+00$ & 1455476 & 1.E+00 \\
\hline & 1.64E-015.92E+01 & 1.38E-017.17E- & & $128.70 \mathrm{E}+00$ & 1457207 & 1.E+00 \\
\hline & 1.63E-015.96E+01 & 1.35E-017.19E- & & $128.80 \mathrm{E}+00$ & 1458922 & 1.E+00 \\
\hline & 1.62E-016.00E+01 & 1.33E-017.21E-01 & & 3.E+01 & 1.0 & 1.E+00 \\
\hline 9.E-02 & $9 . E+01$ & 2.04E-02842E-01 & & 4.E+01 & 2.0 & 5.E-01 \\
\hline 9.E-02 & 9.E+01 & 1.96E-02844E-01 & & 4.E+01 & 2.0 & 5.E-01 \\
\hline 8.E-02 & 9.E+01 & 1.58E-02849E-01 & & 4.E+01 & 2.0 & 5.E-01 \\
\hline 8.E-02 & $9 . E+01$ & 1.51E-028.50E-01 & & 4.E+01 & 2.0 & 5.E-01 \\
\hline 8.E-02 & 9.E+01 & 1.44E-028.51E-01 & & 4.E+01 & 2.0 & 5.E-01 \\
\hline 5.E-02 & 1.E+02 & 1.E-03 & & 8.68E-013.81E+01 & 2.0 & 2.E-01 \\
\hline $5 . \mathrm{E}-02$ & 1.E+02 & 1.E-03 & & 8.68E-013.81E+01 & 2.0 & 2.E-01 \\
\hline 5.E-02 & 1.E+02 & 7.42E-02 & 9.E-01 & 4.E+01 & 2.0 & 2.E-01 \\
\hline 5.E-02 & 1.E+02 & 5.E-04 & & 8.68E-013.82E+01 & 2. & 2.E-01 \\
\hline 5.E-02 & 1.E+02 & 2.E-04 & & 8.68E-013.82E+01 & 2. & 2.E-01 \\
\hline $0.00 \mathrm{E}+00$ & & $0.00 E+00$ & & & & $0.00 E+00$ \\
\hline
\end{tabular}

Table 2: The envelope solution and the core solution.

$$
U_{f}=3-\frac{3}{10} \eta_{f}^{2}+\ldots \text { and } V_{f}=\frac{5 \eta_{f}^{2}}{6}\left(1+\frac{\eta_{f}^{2}}{60}+. .\right)
$$

However, we yet to find the luminosity and the radius of star. From equation (26) we have

$$
C=\frac{3 \kappa_{0}}{256 \pi^{3} a c} \times\left(\frac{1}{G}\right)^{7.5} \frac{L R^{0.5}}{M^{5.5}} \times\left(\frac{R}{\mu}\right)^{7.5}
$$

And from equation

$$
D=\frac{\varepsilon_{0} X X_{C N}}{4 \times 10^{96} \pi} \times(\mu \mathrm{G}) \cdot\left(\frac{1}{R}\right)^{16} \times \frac{M^{18}}{L R^{19}}
$$

Eliminating $\mathrm{L}$ from above equation we have

$$
R^{18.5}=\frac{3 \kappa \varepsilon_{0} X X_{C N}(\mu \mathrm{G})^{8.5} M^{12.5}}{1024 \times 10^{96} \pi 4 C D a c} \times\left(\frac{1}{R}\right)^{8.5}
$$

Substituting all the values of the constants and parameters in equation (37) we have, $R=1.5011 R_{\oplus}$. And using this value of $R$ in equation (38) we have the value of $\mathrm{L}$, that is, $L=6.4957 \mathrm{~L}$.

\section{Conclusion}

In this paper we have assumed a non-rotating and non-magnetic star with mass $2.5 \mathrm{M}_{\AA}$. The structure of the star with Kramer's opacity with negligible abundances heavy element i.e., the pressure, temperature, mass, luminosity and density at various interior point are determined numerically and non-dimensional result of the radiative envelope are shown in Table 1 and convective core in Table 2. However, the complete structure is shown in Table 3 . We also determined the actual radius $R=1.5011 R_{\AA}$ and total luminosity $L=6.4957 L_{\AA}$. And our 
Citation: Bin Masud A (2017) A Study of Stellar Model with Cramer's Opacity by using Runge Kutta Method with Programming C. J Phys Math 8: 239. doi: 10.4172/2090-0902.1000239

Page 8 of 8

\begin{tabular}{|c|c|c|c|c|c|c|}
\hline $\mathbf{x}$ & $\mathbf{P}$ & $\mathbf{Q}$ & $T$ & $\mathbf{P}$ & $\log p$ & L \\
\hline \multirow[t]{2}{*}{$1.00 \mathrm{E}+00$} & $0.00 \mathrm{E}+00$ & $1.00 \mathrm{E}+00$ & $0.00 E+00$ & & & \\
\hline & & & & $5.12 \mathrm{E}-$ & - & \\
\hline \multirow[t]{2}{*}{ 1. $E+00$} & 4.E-09 & $1.00 \mathrm{E}+00$ & 2.E-03 & 7 & & $6.290871 .00 \mathrm{E}+00$ \\
\hline & & & & 2.45E- & - & \\
\hline \multirow[t]{2}{*}{ 9.E-01 } & & 1.13E-051.00E+00 & 2.E-02 & 4 & & $3.610641 .00 \mathrm{E}+00$ \\
\hline & & & & 2.53E- & - & \\
\hline \multirow[t]{2}{*}{ 9.E-01 } & & 2.38E-041.00E+00 & 3.E-02 & 3 & & $2.597571 .00 \mathrm{E}+00$ \\
\hline & & & & $1.16 \mathrm{E}-$ & - & \\
\hline \multirow[t]{2}{*}{ 8.E-01 } & 2.E-03 & 1.E+00 & $5 . E-02$ & 2 & 2 & $1.00 \mathrm{E}+00$ \\
\hline & & & & 3.74E- & - & \\
\hline 8.E-01 & 8.E-03 & 1. $E+00$ & 7.E-02 & 2 & 142662 & $1.00 \mathrm{E}+00$ \\
\hline
\end{tabular}

Table 3: The Convective structure of the model star for $M=2.5, X=0.90, Y=0.09, Z=0.01$.

calculated results are in good agreement with the recent published results book Bohm-Vitense (W. Brunish). If the mass varies and composition fixed, then Teff and Rare found to varies but $\mathrm{L}$ is increase quite sharp. Again if hydrogen and heavy elements are increase, then $\mathrm{R}$ is increase but decrease $\mathrm{L}$ and Teff. For an increase in $\mathrm{M}$ the position of the star in the HR diagram is slightly shifted to toward the upper end of the main sequence. If the mass is constant then a decrease in the hydrogen content of the star increases luminosity and effective temperature. But as time goes on in the main sequence lifetime of a star its hydrogen content gradually diminishes giving rise to the helium content. That means, as a main sequence star ages its position in the HR diagram slowly moves along the main sequence toward the hot end.

\section{References}

1. Bohm-Vitense E (1992) Introduction to Stellar Astrophysics. Cambridge University Press, UK

2. Cowling TG (1936) Remarks on L. Biermann's paper »Konvektion im Innern der Sterne. Astronomische Nachrichten 258: 133-134

3. Schwarzschild M and Härm R (1965) Thermal Instability in Non-Degenerate (low mass) Stars. The Astrophysical Journal 142: 855.

4. Henyey LG, WiletsL, Böhm KH, LeLevier R, Levee RD (1959) A Method for Automatic Computation of Stellar Evolution. The Astrophysical Journal 129: 628. 